\title{
Psychological outcomes related to exome and genome sequencing result disclosure: a meta-analysis of seven Clinical Sequencing Exploratory Research (CSER) Consortium studies
}

\author{
Jill O. Robinson, $\mathrm{MA}^{1}$, Julia Wynn, $\mathrm{MS}{ }^{2}{ }^{2}$, Barbara Biesecker, $\mathrm{PhD}, \mathrm{MS}^{3}$, Leslie G. Biesecker, $\mathrm{MD}^{4}$, \\ Barbara Bernhardt, $\mathrm{MS}^{5}$, Kyle B. Brothers, MD, PhD ${ }^{6}$, Wendy K. Chung, MD, PhD ${ }^{2,7}$, \\ Kurt D. Christensen, PhD ${ }^{8}{ }^{\text {, }}$, Robert C. Green, MD, MPH (1) ${ }^{8,9}$, Amy L. McGuire, JD, PhD ${ }^{1}$, \\ M. Ragan Hart, MS, PhD ${ }^{10}$, Ida Griesemer, MSPH (1) ${ }^{11}$, Donald L. Patrick, MSPH, PhD ${ }^{12}$, \\ Christine Rini, PhD ${ }^{13,14}$, David Veenstra, PharmD, PhD ${ }^{15,16}$, Angel M. Cronin, MS ${ }^{17}$ and \\ Stacy W. Gray, MD, AM 18,19
}

\begin{abstract}
Purpose: As exome and genome sequencing (ES/GS) enters the clinic, there is an urgent need to understand the psychological effects of test result disclosure. Through a Clinical Sequencing Exploratory Research (CSER), phase 1 (CSER1) Consortium collaboration, we evaluated participants' psychological outcomes across multiple clinical settings.

Methods: We conducted a random effects meta-analysis of state anxiety (Hospital Anxiety and Depression Scale [HADS]/Generalized Anxiety Disorder 7-item), depressive symptoms (HADS/ Personal Health Questionnaire 9-item), and multidimensional impact (i.e., test-related distress, uncertainty and positive impact: modified Multidimensional Impact of Cancer Risk Assessment/ Feelings About Genomic Testing Results scale).
\end{abstract}

Results: Anxiety and depression did not increase significantly following test result disclosure. Meta-analyses examining mean differences from pre- to postdisclosure revealed an overall trend for a decrease in participants' anxiety. We observed low levels of testrelated distress and perceptions of uncertainty in some populations (e.g., pediatric patients) and a wide range of positive responses.

Conclusion: Our findings across multiple clinical settings suggest no clinically significant psychological harms from the return of ES/ GS results. Some populations may experience low levels of testrelated distress or greater positive psychological effects. Future research should further investigate the reasons for test-related psychological response variation.

Genetics in Medicine (2019) 21:2781-2790; https://doi.org/10.1038/s41436019-0565-3

Keywords: exome sequencing; genome sequencing; return of results; patient-reported outcomes; psychological effect

\section{INTRODUCTION}

Exome and genome sequencing (ES/GS) promises to transform clinical research and health care. ES and GS can enable researchers and clinicians to identify disease causes, tailor treatments, determine carrier status, and refine disease risk estimates. However, ES/GS presents novel challenges in research and clinical care because it generates vast amounts of data that are, at times, highly uncertain and can reveal information that may or may not have direct bearing on the clinical context in which the sequencing is being performed. While studies have shown that most participants want to learn ES/GS information, there are concerns within the research community about the potential effects that this type of information may have on individuals. ${ }^{1-6}$ It is unclear whether the benefits and utility of returning genomic information to individuals outweigh the potential risks of psychological harms.

\footnotetext{
${ }^{1}$ Center for Medical Ethics and Health Policy, Baylor College of Medicine, Houston, TX, USA; ${ }^{2}$ Department of Pediatrics, Columbia University Medical Center, New York, NY, USA ${ }^{3}$ RTI, International D.C. Office, Washington, DC, USA; ${ }^{4}$ Medical Genomics and Metabolic Genetics Branch, National Human Genome Research Institute, Bethesda, MD, USA; ${ }^{5}$ Division of Translational Medicine and Human Genetics, Perelman School of Medicine at the University of Pennsylvania, Philadelphia, PA, USA; ${ }^{6}$ Department of Pediatrics, University of Louisville School of Medicine, Louisville, KY, USA; ${ }^{7}$ Department of Medicine, Columbia University Medical Center, New York, NY, USA; ${ }^{8}$ Department of Medicine, Brigham and Women's Hospital and Harvard Medical School, Boston, MA, USA; ${ }^{9}$ Broad Institute of MIT, Cambridge, MA, USA; ${ }^{10}$ Department of Biomedical Data Science, Stanford University, Stanford, CA, USA; ${ }^{11}$ Department of Health Behavior, University of North Carolina, Chapel Hill, NC, USA; ${ }^{12}$ Department of Health Services, University of Washington, Seattle, WA, USA; ${ }^{13}$ John Theurer Cancer Center, Hackensack University Medical Center, Hackensack, NJ, USA $;{ }^{14}$ Georgetown University School of Medicine, Washington, DC, USA; ${ }^{15}$ Clinical Sequencing Exploratory Research Coordinating Center, University of Washington, Seattle, WA, USA; ${ }^{16}$ Department of Pharmacy, University of Washington, Seattle, WA, USA $;{ }^{17}$ Corrona, LLC, Waltham, MA, USA $;{ }^{18}$ Department of Population Science, City of Hope, Duarte, CA, USA; ${ }^{19}$ Department of Medical Oncology \& Therapeutics Research, City of Hope, Duarte, CA, USA. Correspondence: Stacy W. Gray (stagray@coh.org)

Co-first authors: Jill O. Robinson and Julia Wynn
}

Submitted 1 February 2019; accepted: 22 May 2019

Published online: 13 June 2019 
Previous systematic reviews on the psychological outcomes of genetic testing in the context of adult and pediatric single or multigene testing for diseases such as cancer, Alzheimer disease, and Huntington disease have shown that, in general, test result disclosure does not cause depression or anxiety. ${ }^{8-11}$ When negative psychological effects are observed, they are often short-lived. ${ }^{8,11}$ Limited data are available on the psychological effects of the disclosure of ES/GS results. However, several studies have found that there may be a subset of individuals who may be more likely to experience higher test-related distress. A study of parents whose children had diagnostic ES $(n=192)$ demonstrated that parents of tested children experienced psychological responses to testing including worry, fear, and relief with test-related distress and uncertainty higher among those whose children received a genetic diagnosis. ${ }^{12} \mathrm{~A}$ longitudinal cohort study of individuals undergoing GS $(n=35)$ noted that test distress was low and that most participants were happy or relieved about their results. $^{13}$

To more completely explore the effects of the clinical integration of ES/GS across multiple clinical contexts and patient populations, the National Human Genome Research Institute (NHGRI) launched the Clinical Sequencing Exploratory Research (phase 1; CSER1) Consortium. ${ }^{14}$ The CSER1 Consortium studies returned results from ES/GS to a range of participants including parents of children with rare diseases, suspected genetic conditions, and cancer; healthy adults (including those at high risk of developing cancer); and adults with diseases such as cancer and cardiomyopathy. CSER1 investigators identified high priority areas of investigation at the consortium's inception and coordinated outcome measurement across sites to facilitate cross-consortium analyses. ${ }^{15}$ Within the psychosocial impact domain, investigators coordinated the measurement of anxiety, depression, and multidimensional psychological impact across several sites, as these outcomes have been commonly assessed in prior studies of genetic testing. ${ }^{16}$ Our overarching objective was to conduct a meta-analysis across CSER1 studies to assess state anxiety, depressive symptoms, and multidimensional test-related outcomes following the return of ES/GS results.

\section{CSER study sites}

\section{MATERIALS AND METHODS}

Participating CSER studies ${ }^{14}$ included Brigham and Women's Hospital and Harvard Medical School's MedSeq Project, Children's Hospital of Philadelphia's PediSeq study, Columbia University Medical Center, Dana-Farber Cancer Institute and Broad Institute's (DFCI) CanSeq study, NHGRI's ClinSeq ${ }^{\circledR}$ study, the University of North Carolina's NCGENES study, and the University of Washington's New EXome Technology in (NEXT) Medicine study. A detailed description of the individual study protocols can be found in primary publications from each site and a summary is presented in Table $1 .^{14,15}$ Informed consent was obtained from all participants and each site gathered and shared data with local Institutional Review Board (IRB) approval. Each CSER site developed policies and frameworks for the disclosure of primary diagnostic and secondary findings, thus the type of results disclosed differed across sites. ${ }^{17}$ Sites conducted disclosure sessions in person, with the exception of Columbia, which conducted disclosures both in person and via video conference. Sequencing, variant interpretation, disclosure of results, and administration of participant questionnaires were performed at individual CSER sites from June 2015 to August 2017, and the collected data were combined for post hoc analyses.

\section{Measures}

State anxiety and depressive symptoms were measured preand post-ES/GS results disclosure using the Hospital Anxiety and Depression Scale (HADS) ${ }^{18}$ (three sites), the Personal Health Questionnaire 9-item (PHQ-9) ${ }^{19}$ (three sites), and the Generalized Anxiety Disorder 7-item (GAD-7) ${ }^{20}$ (two sites). The multidimensional impact of receiving ES/GS results was measured following results disclosure using a modified version of the Multidimensional Impact of Cancer Risk Assessment (MICRA) ${ }^{21}$ (six sites) or the Feelings About genomiC Testing Results (FACToR) $)^{22}$ (two sites). These scales are similar, with comparable subscales assessing the same time period (past week). The measures differ somewhat in terms of the question stems and subscale wording. The MICRA evaluates test-related distress ("Feeling upset about my test results"), uncertainty ("Being uncertain about what my test result means about my cancer risk"), and positive experiences (reverse scored) ("Feeling relieved about my test results") while the comparable subscales in the FACToR assess negative emotions ("How upset did you feel about your genetic test result?"), uncertainty ("How uncertain did you feel about what your genetic test results mean for you?"), and positive feelings ("How happy did you feel about your genetic test results?"). The wording for the MICRA relates to frequency while the FACToR relates to intensity. Response option anchors ranged from "Never" to "Often" for the MICRA and "Not at all" to "A great deal" for the FACToR. The MICRA scale was not uniformly modified across the six sites administering it. The original scale used the phrase "my test results" and some sites differed in how they made modifications to identify the specific results in their study such as "my genomic screening results" or "my genetic test results." Additionally, five questions in the original scale specifically referenced cancer and modification of these questions to address ES/GS was variable across sites. Finally, one site dropped an item in the MICRA that queried participants about "having difficulty making decisions about cancer screening or prevention" as such items are not relevant to participants living with advanced cancer. Responses on the baseline and the first completed postdisclosure survey were analyzed. Missing data were imputed using the last observation carrier backward; if a participant completed a survey at the time of the second postdisclosure survey, but not at the time of the first postdisclosure survey, then the participant's responses at the time of the second postdisclosure survey were 


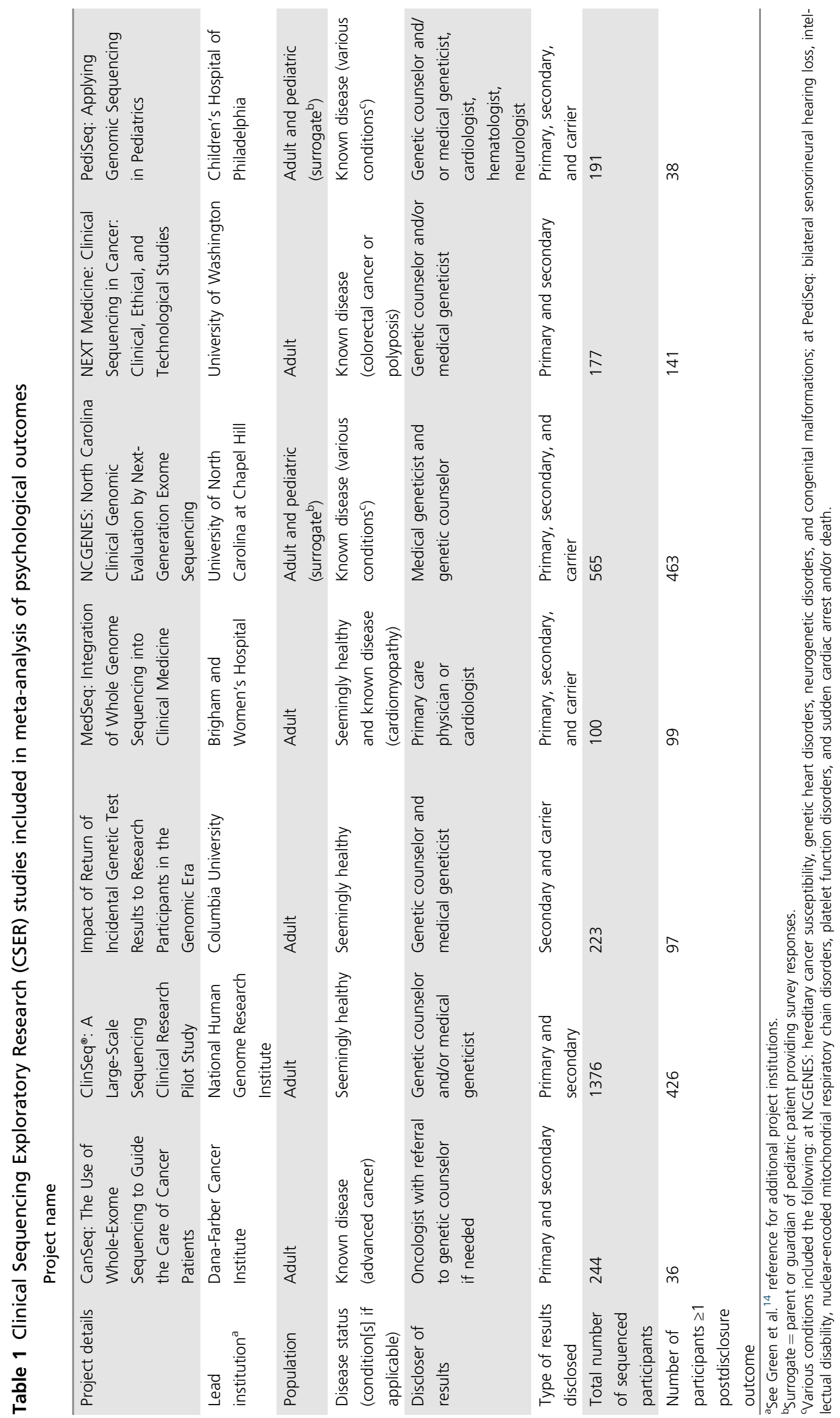


included. Median time between return of results to first postdisclosure survey was 24 days (interquartile range [IQR] 14-37 days, range 1-396 days), and median time between return of results to second postdisclosure survey was 184 days (IQR 142-206 days, range 54-445 days). The majority of participants' (96\%) responses included in this analysis are from the first postdisclosure survey. Participants who dropped out of the study prior to results disclosure were omitted from analyses.

\section{Statistical methods}

For state anxiety (HADS-Anxiety [A] and GAD-7) and depressive symptoms (HADS-Depression [D] and PHQ-9), we described the site-specific predisclosure and postdisclosure values and absolute change (postdisclosure minus predisclosure). For the multidimensional impact of testing (MICRA and FACToR), we described the site-specific postdisclosure values for each subscale. For all outcomes, we conducted random effects meta-analyses using the method of DerSimonian and Laird. ${ }^{23}$ We specified the random effects model a priori, since we expected the effect size to vary between sites due to the heterogeneity in study designs and participant characteristics by site. In a random effects model, the weight of each study is derived from both (1) within-study variability (i.e., the number of participants in the study) and (2) between-study variability (i.e., the difference in effects between the studies). Thus, the combined effect size is not dominated by a single study with a large number of participants. For the meta-analyses, we calculated standardized mean differences in pre- and post-results disclosure state anxiety (HADS-A and GAD-7) and depressive symptoms (HADS-D and PHQ-9). To examine responses from the different measures and across studies, we rescaled the subscale scores of the multidimensional impact of testing (MICRA and FACToR) to a 0-100 point scale, where 0 represents "Not at all" or "Never" and 100 represents "A great deal" or "Often." Given the variations of modifications made to the MICRA and FACToR scales, as well as differences in study populations and sequencing goals, we focused the meta-analyses on identifying broad trends rather than statistical comparisons. In exploratory analyses, we evaluated the differences in postdisclosure outcomes for (1) those participants who had and had not received any type of genetic results returned as part of the study, (2) those participants who received primary results, and (3) those participants who received carrier and/or pharmacogenetic results. Statistical analyses were conducted with Stata version $13.1 .^{24}$

\section{RESULTS}

Across seven CSER1 studies, 2876 participants were sequenced, and 1300 participants reported at least one postdisclosure outcome that was included in this metaanalysis (multidimensional impact $n=1299$, anxiety $n=741$, and depressive symptoms $n=850$, Table 1). Participant characteristics and information about types of ES/GS results returned by site are shown in Table 2 . Studies varied by whether the testing was used for diagnostic purposes, and all sites returned at least some secondary findings to participants.

\section{Anxiety and depressive symptoms}

Figure 1 displays the pre- and postdisclosure anxiety (HADS and GAD-7) and depressive symptoms (HADS and PHQ-9) responses, as well as the absolute change over time. The majority of participants across sites were below the threshold for clinical anxiety and depression at baseline, using validated cutoffs for the measures. ${ }^{18-20}$ We observed no statistically significant changes in anxiety and depressive symptoms following return of ES/GS results, or in the absolute change (postdisclosure minus predisclosure) (Fig. 1a, b, d, e). Random effects meta-analyses examining mean differences from pre- to postdisclosure revealed an overall trend of decrease in anxiety among participants, with the greatest decreases noted in participants from NCGENES and MedSeq (effect $[95 \% \mathrm{CI}]:-0.36[-0.45,-0.26],-0.52[-0.73$, $-0.30]$, respectively, Fig. 1c). We identified a similar trend of decrease in depressive symptoms across sites, with the greatest decrease noted among participants at Columbia, MedSeq, and NCGENES (effect [95\% CI]: $-0.33[-0.53$, $-0.13],-0.22 \quad[-0.40,-0.04],-0.16 \quad[-0.26,-0.07]$, respectively, Fig. 1f).

\section{Negative emotional impact and uncertainty}

Using two measures to assess the multidimensional impact of receiving ES/GS results (MICRA and FACToR), in general, we identified low levels of postdisclosure test-related negative emotions and uncertainty, although there was some variation across sites (Fig. 2a-f). Negative emotions and distress were generally infrequent, with more variability and somewhat greater reported distress among participants at the two sites that included pediatric populations, PediSeq and NCGENES, relative to the other sites (Fig. 2a, b). Lowest levels of distress were observed in Columbia, MedSeq, and ClinSeq ${ }^{\circledR}$ (effect [95\% CI]: 1.46 [0.41, 2.50], 2.27 [1.18, 3.37], 4.26 [3.33, 5.18], respectively, Fig. 2c), all of which enrolled seemingly healthy adults or adults with cardiovascular disease.

Responses to the test-related uncertainty subscale varied across studies, with participants overall reporting low levels of postdisclosure uncertainty. Uncertainty was higher among the studies that included parents of pediatric patients (PediSeq, effect [(95\% CI]: $23.22[17.11,29.34]$ and NCGENES, 22.17 [20.31, 24.03]) compared with the studies of mostly healthy adults (effect [95\% CI]: Columbia, 3.58 [1.98, 5.17]; MedSeq, 6.72 [3.97, 9.47], ClinSeq $^{\circledR}, 7.99$ [6.89, 9.09]), while uncertainty was more intermediate for NEXT Medicine (effect [95\% CI]: $15.89[12.69,19.08])$, a study of adults with colon polyps or cancer (Fig. 2f).

\section{Positive emotional impact}

Across sites we saw the widest range of responses to the positive emotions subscales (Fig. 2g-i). Descriptive analyses showed that PediSeq reported positive responses from all participants $(n=38)$, whereas all other studies had much 
Table 2 Participant characteristics by CSER study site

\begin{tabular}{|c|c|c|c|c|c|}
\hline $\begin{array}{l}\text { CanSeq } \\
N=36\end{array}$ & $\begin{array}{l}\text { ClinSeq }^{\circledR} \\
N=426\end{array}$ & $\begin{array}{l}\text { Columbia } \\
N=97\end{array}$ & $\begin{array}{l}\text { MedSeq } \\
N=99\end{array}$ & $\begin{array}{l}\text { NCGENES } \\
N=463\end{array}$ & $\begin{array}{l}\text { NEXT } \\
\text { Medicine } \\
N=141\end{array}$ \\
\hline
\end{tabular}

\begin{tabular}{|c|c|c|c|c|c|c|c|}
\hline \multicolumn{8}{|l|}{ Age } \\
\hline Median & 58 & 57 & 47 & 56 & 33 & 55 & 37 \\
\hline IQR & $51-63$ & 53-61 & $38-58$ & $48-63$ & $9-53$ & 44-61 & $32-40$ \\
\hline Range & $31-81$ & $45-65$ & $20-82$ & $18-84$ & $0-84$ & $24-82$ & $24-41$ \\
\hline \multicolumn{8}{|l|}{ Gender, \% } \\
\hline Male & 44 & 53 & 30 & 47 & 40 & 47 & 61 \\
\hline Female & 56 & 47 & 70 & 53 & 60 & 53 & 39 \\
\hline \multicolumn{8}{|l|}{ Ethnicity, \% } \\
\hline Non-Hispanic & 97 & 98 & 95 & 93 & 93 & 98 & 97 \\
\hline Hispanic & 3 & 2 & 5 & 5 & 6 & 2 & 3 \\
\hline Unknown & 0 & 0 & 0 & 2 & 1 & 0 & 0 \\
\hline \multicolumn{8}{|l|}{ Race, \% } \\
\hline White & 81 & 92 & 89 & 91 & 83 & 85 & 76 \\
\hline Black & 8 & 1 & 3 & 3 & 12 & 0 & 5 \\
\hline Other $^{a}$ & 6 & 5 & 4 & 4 & 1 & 15 & 13 \\
\hline Unknown & 6 & 1 & 4 & 2 & 3 & 0 & 5 \\
\hline \multicolumn{8}{|l|}{ Surrogate, $\%{ }^{b}$} \\
\hline No & 100 & 100 & 100 & 100 & 61 & 100 & 0 \\
\hline Yes & 0 & 0 & 0 & 0 & 39 & 0 & 21 \\
\hline Unknown or NA & 0 & 0 & 0 & 0 & 0 & 0 & 79 \\
\hline \multicolumn{8}{|l|}{ Marital status, \% } \\
\hline Married & 72 & 75 & 91 & 68 & 67 & 68 & 11 \\
\hline Not married (adult) & 28 & 21 & 9 & 32 & 33 & 32 & 11 \\
\hline Unknown or NA & 0 & 4 & 0 & 0 & 0 & 0 & 79 \\
\hline \multicolumn{8}{|l|}{ Education, \% } \\
\hline High school or less & 17 & 4 & 8 & 6 & 18 & 16 & 8 \\
\hline Some college & 22 & 6 & 20 & 10 & 40 & 22 & 5 \\
\hline College degree & 25 & 26 & 31 & 33 & 25 & 39 & 5 \\
\hline More than college & 33 & 60 & 41 & 51 & 16 & 23 & 3 \\
\hline Unknown & 3 & 3 & 0 & 0 & 0 & 0 & 79 \\
\hline \multicolumn{8}{|l|}{ Sequencing, \% } \\
\hline Germline only & 0 & 100 & 100 & 100 & 100 & 96 & 100 \\
\hline Germline + somatic & 100 & 0 & 0 & 0 & 0 & 4 & 0 \\
\hline \multicolumn{8}{|c|}{ Return of any result, \% } \\
\hline No & 0 & 15 & 0 & 0 & 58 & 25 & 16 \\
\hline Yes & 100 & 85 & 100 & 100 & 42 & 75 & 84 \\
\hline \multicolumn{8}{|c|}{ Return of secondary findings, \% } \\
\hline No & 83 & 15 & 0 & 0 & 91 & 30 & 34 \\
\hline Yes & 17 & 85 & 100 & 100 & 9 & 70 & 66 \\
\hline \multicolumn{8}{|c|}{ Diagnostic category, \% } \\
\hline Healthy individuals & - & 80 & 100 & 51 & - & - & - \\
\hline $\begin{array}{l}\text { Cardiovascular } \\
\text { disease }\end{array}$ & - & 20 & - & 49 & 9 & - & 26 \\
\hline $\mathrm{DD} / \mathrm{ID}$ & - & - & - & - & 28 & - & 16 \\
\hline Hereditary cancer & - & - & - & - & 21 & 100 & - \\
\hline Neuromuscular & - & - & - & - & 20 & - & 5 \\
\hline Ophtho/retina & - & - & - & - & 8 & - & - \\
\hline Cancer & 100 & - & - & - & - & - & - \\
\hline Other & - & - & - & - & 13 & - & 53 \\
\hline
\end{tabular}

CSER Clinical Sequencing Exploratory Research Consortium, DD developmental delay, ID intellectual disability, IQR interquartile range, Ophtho ophthalmology disorder.

ather includes Asian, $n=26$; Native American, $n=3$; and multiracial, $n=19$.

${ }^{\mathrm{b}}$ Surrogate $=$ parent or guardian of pediatric patient providing survey responses.

more variability, with responses ranging from 0 to the higher end of the scale (Fig. $\mathbf{2 g}$, h). Aligned with the descriptive data, the random effects meta-analysis revealed a large range of positive experiences across sites. There was no pattern to the frequency of positive responses by study type, with the most positive responses observed among participants at the PediSeq, MedSeq, and Columbia sites (effect [95\% CI]: $58.92[50.94,66.89], 56.45[50.26,62.63], 56.29$ [49.54, 63.04], respectively) and least positive responses observed at NCGENES, ClinSeq ${ }^{\circledR}$, and DFCI (effect [95\% CI]: 32.38 [30.01, 34.75], 38.50 [35.73, 41.27], 39.85 [28.01, 51.69], respectively, Fig. 2i).

In exploratory meta-analyses, comparing participants who had received any type of result (e.g., primary, secondary, or carrier) returned in the study and those who did not receive any study results, we did not observe differences in psychological outcomes (Supplemental Figs. 1-6). When examining outcomes by result group (primary or carrier/ pharmacogenetic; Supplemental Figs. 7-26), mean differences from pre- to postdisclosure revealed an overall trend of decrease in anxiety and depression among participants of both groups (Supplemental Figs 7, 8). However, among participants who received primary findings, as compared with carrier/pharmacogenetic findings, there was higher testrelated distress (10.47 [3.33, 17.62]: $3.61 \quad[1.98, \quad 5.24]$ respectively) and uncertainty (22.92 [9.59, 36.26]: 10.44 $[6.77,14.11]$ respectively). We did not perform an analysis of the group of participants who only received secondary results because the return of secondary results without the return of primary results was uncommon. 

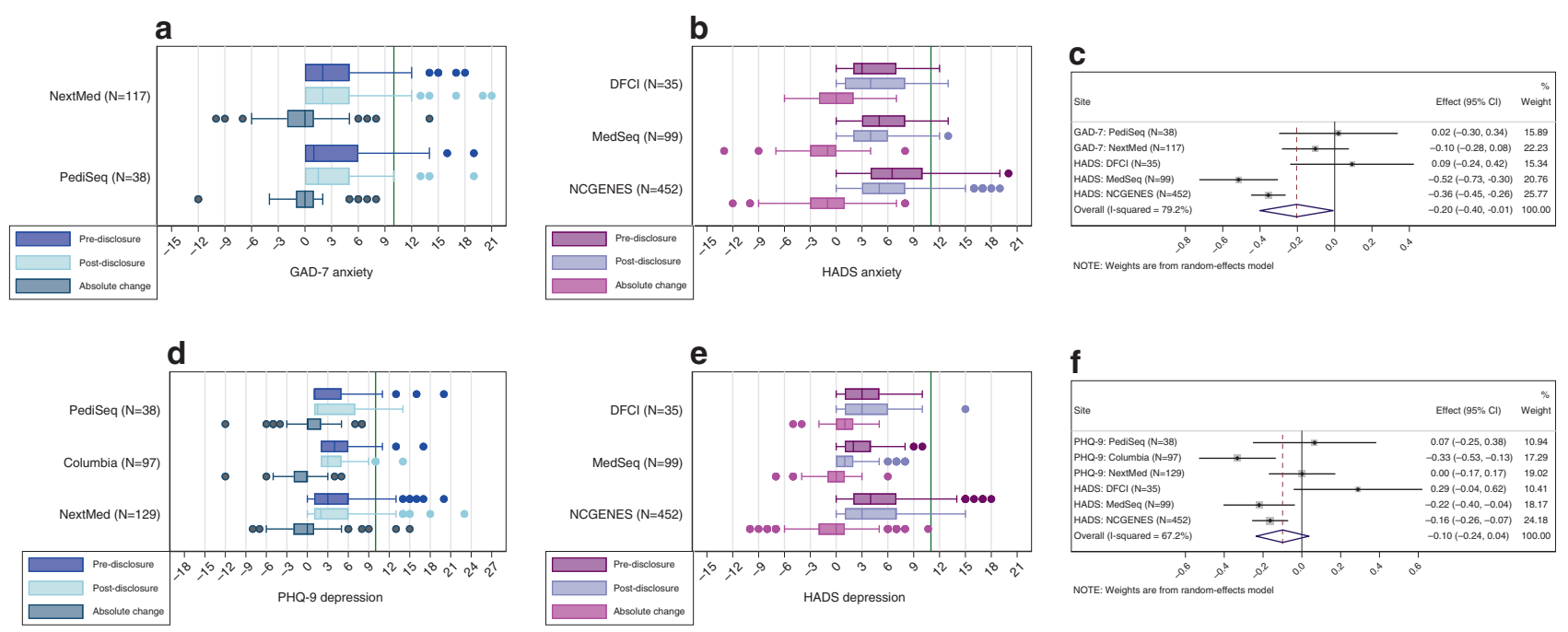

Fig. 1 Box and whisker plots of participants' anxiety (a GAD-7; b HADS) and depressive symptoms (d PHQ-9; e HADS) before (pre) and after (post) disclosure of genome sequencing results, as well as forest plots for absolute change over time (c change in GAD-7/HADS anxiety; $\mathbf{f}$ change in PHQ-9/HADS depression). Clinical level of anxiety and depression is shown with the green line. GAD-7 Generalized Anxiety Disorder 7-item, HADS Hospital Anxiety and Depression Scale, PHQ-9 Personal Health Questionnaire 9-item.

a

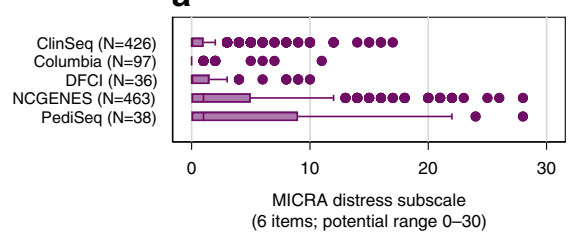

d

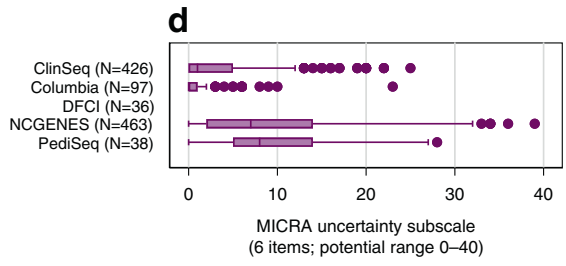

g

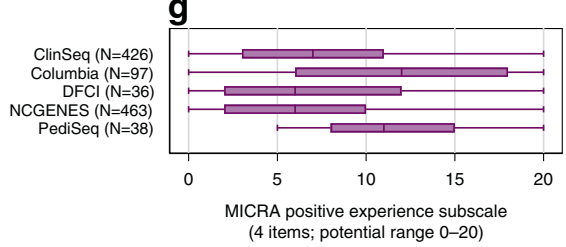

b
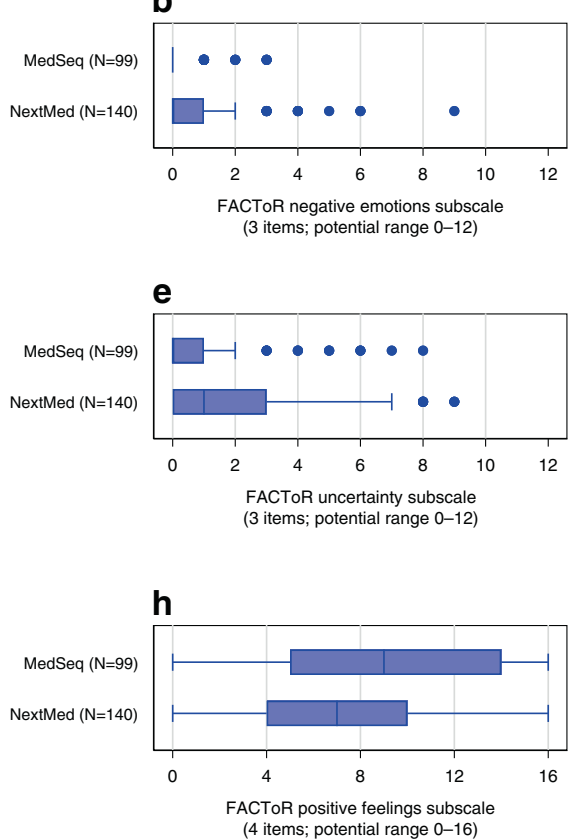

C

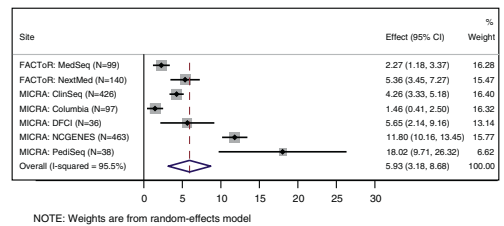

f

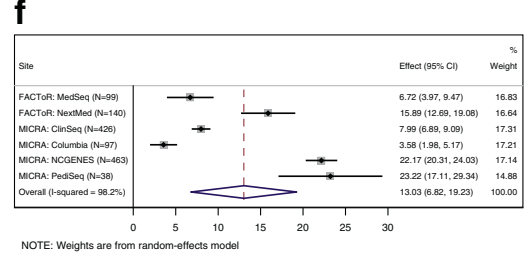

i

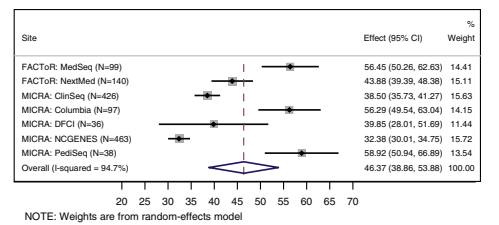

Fig. 2 Box and whisker and forest plots of participants' multi-dimensional impact of receiving genome sequencing results including test-related negative emotions/distress, uncertainty, and positive experiences ( $\mathbf{a}, \mathbf{d}, \mathbf{g}$ MICRA; b, e, h FACToR; $\mathbf{c}, \mathbf{f}, \mathbf{i}$ MICRA/FACToR). MICRA Multidimensional Impact of Cancer Risk Assessment FACTOR Feelings About genomiC Testing Results.

\section{DISCUSSION}

Our meta-analysis of psychological outcomes from seven CSER1 Consortium studies with a total of 1300 sequenced participants who reported at least one postdisclosure outcome represents, to our knowledge, the largest examination of psychological responses following receipt of ES/GS information to date. The majority of participants in the represented CSER1 studies did not experience a significant increase in state anxiety or depression following ES/GS test result disclosure. Additionally, most participants scored below the thresholds for clinically significant anxiety and depression both before and after testing. Meta-analyses examining mean differences from pre- to postdisclosure also revealed an overall trend of a decrease in participants' anxiety. Furthermore, when examining test-related psychological outcomes specifically, participants' responses were generally neutral to positive. However, we also identified modest levels of testrelated distress and uncertainty in some populations. Our 
findings are consistent with other studies and strengthen the evidence that, for the majority of participants, there are negligible to no psychological harms related to receiving results from ES/GS. ${ }^{13,25,26}$

Despite the fact that overall psychological distress was minimal for participants receiving ES/GS results, we observed some variation in psychological responses for different patient populations following test result disclosure and there are several trends in the data worth investigating in future studies. First, we saw that test-related uncertainty and distress were lower in studies that included healthy adults $\left(\mathrm{ClinSeq}^{\circledR}\right.$, Columbia, and MedSeq), intermediate in studies of adults with known disease (NextMed, DCFI), and greatest among studies of children with known disease (PediSeq, and NCGENES, the latter of which also included adults with known disease). These data raise the question of whether adults with a known disease, and particularly parents of children with a known disease, may be more prone to negative test-related psychological experiences following ES/GS than healthy individuals. Healthy adults may also have demonstrated lower distress or uncertainty because they were relatively healthy, voluntary participants of a sequencing study where they would learn their genomic health risk information and few received secondary findings indicating disease risk. Furthermore, although the small number of patients reporting postdisclosure outcomes in the DFCI cohort limits generalizability and may reflect ascertainment bias (lower postdisclosure survey completion rates due, in part, to illness or death), it was notable that the psychological responses to ES observed at this site were somewhat more modest, and less distressed, than the PediSeq and NCGENES cohorts. One might hypothesize that the relative lack of testrelated distress among this population may be reflective of the severity of the participants' disease and the fact that germline disease related genetic testing results infrequently have medical implications for the tested proband and secondary results may be less relevant for someone with advanced cancer. A similar pattern has been reported among women undergoing genetic testing for breast cancer. Studies have demonstrated lower negative experiences (uncertainty or distress) and greater positive responses related to identification of a pathogenic variant among women with a cancer diagnosis compared with those unaffected with cancer. ${ }^{27,28}$

Data from some CSER1 sites also suggested a mixed response to sequencing information, particularly in pediatric contexts. For example, in addition to slightly higher levels of test-related distress and uncertainty, PediSeq study participants also had the highest level of positive experiences. Previous qualitative studies of parents whose children have undergone diagnostic genome sequencing have similarly identified mixed reactions following testing. ${ }^{12,29,30}$ Some parents experience a loss of hope with a new genetic diagnosis and, at the same time, experience positive feelings related to the value of the genetic testing potentially providing the ability to pursue more tailored health care or pursue specific research pathways. Additionally, a qualitative study of parents of pediatric cancer patients from a CSER1 study not included in this analysis found that some parents valued ES information even when it did not provide diagnostic information or impact clinical care. Parents described experiencing psychological benefit because they felt that a lack of diagnostic information provided peace of mind that there was less concern of increased risk for other conditions for their child or others in their family. ${ }^{31}$ These mixed responses could also reflect the complexity of the parental role of an ill child, which may lead one to seek out and value their child's genetic information regardless of any personal negative psychological impact it could bring. ${ }^{30,32}$

Another interesting finding was that, although the Columbia, MedSeq, and $\mathrm{ClinSeq}^{\circledR}$ studies had similar populations (i.e., adults who are seemingly healthy or have cardiovascular disease) findings from the multidimensional measures differed in their positive experiences. Columbia and MedSeq participants expressed higher positive experiences than participants in $\mathrm{ClinSeq}^{\circledR}$. One potential explanation for these findings could relate to study design. In the Columbia and MedSeq studies, participants were recruited through and received results from their personal physicians, rather than study staff, as in ClinSeq ${ }^{\circledR}$. Little is known about how responses to ES/GS outcomes may differ based on the context in which the results are being disclosed or if they differ based on who is delivering ES/GS information.

We identified several factors that we hypothesize may influence the emotional impact of ES/GS results, whether positive, negative, uncertain or mixed. Because of the heterogeneity of our study designs and populations, and measurement strategies, we were limited in our ability to statistically compare across the factors identified, including, but not limited to (1) the probands' disease or condition, (2) the age of the proband and where they are in the trajectory of their disease, (3) whether the result ends a diagnostic odyssey, (4) if the result has direct clinical utility for the proband, (5) if the individual reporting outcomes is the proband or surrogate, (6) the mode of result disclosure (e.g., in person, by phone), (7) the relationship between the proband and the person disclosing ES/GS results, and (8) the type of findings that are reported (e.g., primary, secondary, pharmacogenetic, carrier status). Further research exploring these observed differences will help inform both research and clinical care. For example, how people construe results in the context of their own health or illness may reflect their hopes and expectations, which may ultimately impact feelings of disappointment, happiness, or relief in the wake of testing. Similarly, some ES/GS results may have relatively less of a psychological impact if delivered in person by an individual's health-care provider as compared with delivered by phone by someone on the care team who does not have an existing relationship with the patient.

Given the fact that our data revealed more variation in participants' test-related emotions than it did in state anxiety or depression, future work on psychological outcomes may be most revealing if it focuses on the multidimensional impact of 
sequencing rather than on test-independent state psychological measures. In fact, multiple experts are advocating for a more nuanced, or multidimensional, assessment of outcomes. For example, Biesecker and colleagues have argued that commonly used assessments of psychological wellbeing may not be the most appropriate way to assess a recipient's ability to manage genetic and genomic risk information. ${ }^{33}$ Specifically, state anxiety is context dependent, transient, and not necessarily an indicator of poor psychological wellbeing. Furthermore, although depressive symptoms can signal patient vulnerability and depression, such symptoms may also be an expected part of the process of adapting to learning health information. Many providers concur that if a patient receives a test result revealing an increased risk for a disease, it may be appropriate for them to experience anxiety and that this response may be an indication of engagement and understanding of an existent health threat. ${ }^{34}$ Moreover, mild negative psychological responses, as well as positive responses, could be strong motivators to initiate necessary health behavior change. ${ }^{15,35}$ Given all of these considerations, there is a need to assess subtle, in addition to obvious, psychological impact. One of the most important areas of investigation going forward is the need to continue to develop and refine tools to assess test-related outcomes such as test-specific distress, perceptions of uncertainty, and positive responses. Qualitative research methods are likely to be useful for addressing this challenge, because of their ability to provide rich insight into people's lived experiences and understandings.

\section{Limitations}

One of the greatest strengths and limitations of this study is that it draws on data from multiple studies of ES/GS that were implemented in different clinical contexts with different participant populations. The pooling of participants across seven CSER1 studies resulted in one of the largest studies of psychological responses following receipt of genomic results. However, differences in study design, the timing of survey administration, and variation in outcome measurement limited our ability to pinpoint sources of variation across studies. While validated measures were used, the scales that were used differed across sites. Furthermore, the MICRA scale was modified by most sites, and the modifications were not harmonized because the participant population frequently called for context-specific adaptations. Additionally, the MICRA and FACToR differed in how they assessed the multidimensional impact, both in question wording and response options. These differences limited our ability to interpret the cross-site comparison and may have impacted the reliability and validity of the measures. Therefore we cannot eliminate the possibility that our results may be, in part, a function of variations in outcome measurement rather than the actual impact of the results. Additionally, all participants, particularly those who were affected or had a child affected, may have experienced psychological distress or anxiety unrelated to the study protocol, affecting the ability to measure changes specific to the return of ES/GS results. Furthermore, the harmonized instruments may not have captured subtle psychological changes following receipt of genomic results. While the majority of participants did not experience adverse psychological impacts, some sites described rare instances of a distressed or upset participant. Future studies of these rare negative experiences may help to identify risk factors, which could include a history of or existing psychological condition, that may predict poor outcomes and create interventions to prevent this, as well as provide better evidence to define patient concerns surrounding receipt of this information. Although we included baseline measures of clinical anxiety and depressive symptoms, for which the majority of participants were below the clinical threshold, we did not have information about participants' history of clinical anxiety or depression. Moreover, our exploratory analyses are limited in that we combined participants who received any type of result (e.g., primary diagnostic, secondary, and carrier). Given that there may be substantive psychological differences across different types of results received, these findings need to be interpreted with caution. Finally, the generalizability of study findings is limited by the fact that the studies enrolled predominantly white non-Latino/a, well-educated, and English speaking participants and that many participants had prior experience with genetics through clinical care or prior research participation.

\section{CONCLUSIONS}

Results from our meta-analyses of psychological outcomes from genomic sequencing exemplify the ability of consortium studies to create large samples across multiple clinical contexts and study populations. Although there are limitations to our approach, these findings offer strong evidence for the absence of significant psychological harms following receipt of ES/GS results in the studied populations. To more definitively conclude that any psychological harm associated with ES/GS disclosure is outweighed by the benefits, investigators will need to evaluate psychological outcomes in longitudinal, very large, and diverse (in terms of participant demographics, study setting, and disease status and type) populations. Such efforts are underway through multiple large US-based consortiums including the current phase 2 CSER, ${ }^{36}$ Electronic Medical Records and Genomics (eMERGE), ${ }^{37}$ All of US, ${ }^{38}$ and Newborn Sequencing in Genomic medicine and public HealTh (NSIGHT). ${ }^{39}$ Furthermore, investigators should aim to elucidate the causes of variation in psychological outcomes following ES/GS results disclosure. Importantly, there is a great need for tools that will enable researchers and clinicians to prospectively identify individuals who may be at highest risk of clinically significant distress following testing. Individuals at high risk for test-related distress may need additional genetic counseling and support through the testing process to mitigate harm. In addition, investigators will need to more completely explore the full range of positive psychological responses to receiving ES/GS 
information and evaluate for more nuanced emotional states. To accomplish these goals, the research community will need to continue to develop and standardize sequencing-related tools and outcome measures. Only through continued outcome measure standardization, harmonization, and rigorous assessment will we be able to fully understand the psychological effects of ES/GS results disclosure on research participants and patients.

\section{SUPPLEMENTARY INFORMATION}

The online version of this article (https://doi.org/10.1038/s41436019-0565-3) contains supplementary material, which is available to authorized users.

\section{ACKNOWLEDGEMENTS}

The CSER Consortium was funded by the National Human Genome Research Institute (NHGRI) and the National Cancer Institute $(\mathrm{NCl})$, supporting the following sites: CSER Coordinating Center U01HG007307, NEXT Medicine U01HG006507, MedSeq U01HG006500, PediSeq U01HG006546, DFCI U01HG006492, NCGENES U01HG006487. ClinSeq ${ }^{\circledR}$ is supported by ZIAHG200359 09 and ZIAHG200387 04 from the Intramural Research Program of the NHGRI. The Columbia ROR project was supported by R21 HG006596 (Dr. Appelbaum, principal investigator [PI]), R01 HG006600 (Drs. Chung and Phelan, PIs), P50 HG007257 (Dr. Appelbaum, PI), UL1 TR000040, and UL1 RR024156.

\section{DISCLOSURE}

K.D.C. has received travel funds from Illumina, Inc. S.W.G. serves as a consultant to GRAIL. R.C.G. advises AIA, Applied Therapeutics, Helix, Ohana, OptraHealth, Prudential, Verily, and Veritas, and is cofounder of Genome Medical, Inc. Funding for B. Biesecker's contributions came from the Intramural Research Program of the National Human Genome Research Institute, National Institutes of Health. L.G.B. is a member of Illumina Medical Ethics Advisory Board, receives royalties from Genentech, receives in-kind research support from ArQule, Inc, honoraria from Cold Spring Harbor Press, and is supported by the Intramural Research Program of NHGRI, grant HG200387 04. The other authors declare no conflicts of interest.

Publisher's note: Springer Nature remains neutral with regard to jurisdictional claims in published maps and institutional affiliations.

\section{REFERENCES}

1. National Academies of Sciences, Engineering, and Medicine. Returning individual research results to participants: guidance for a new research paradigm. Washington, DC: The National Academies Press; 2018.

2. Wynn J, Martinez J, Duong J, et al. Research participants' preferences for hypothetical secondary results from genomic research. J Genet Couns. 2017;26:841-851.

3. Appelbaum PS, Fyer A, Klitzman RL, et al. Researchers' views on informed consent for return of secondary results in genomic research. Genet Med. 2015; 17:644-650.

4. Townsend A, Adam S, Birch PH, Lohn Z, Rousseau F, Friedman JM. "I want to know what's in Pandora's Box": comparing stakeholder perspectives on incidental findings in clinical whole genomic sequencing. Am J Med Genet A. 2012;158a:2519-2525.

5. Bollinger JM, Scott J, Dvoskin R, Kaufman D. Public preferences regarding the return of individual genetic research results: findings from a qualitative focus group study. Genet Med. 2012;14:451-457.

6. Rini $C$, Khan CM, Moore E, et al. The who, what, and why of research participants' intentions to request a broad range of secondary findings in a diagnostic genomic sequencing study. Genet Med. 2018;20:760-769.

7. Green RC, Roberts JS, Cupples LA, et al. Disclosure of APOE genotype for risk of Alzheimer's disease. N Engl J Med. 2009;361:245-254.

8. Heshka JT, Palleschi C, Howley H, Wilson B, Wells PS. A systematic review of perceived risks, psychological and behavioral impacts of genetic testing. Genet Med. 2008;10:19-32.

9. Peterson EB, Chou WS, Gaysynsky A, et al. Communication of cancerrelated genetic and genomic information: a landscape analysis of reviews. Transl Behav Med. 2018;8:59-70.

10. Crozier S, Robertson N, Dale M. The psychological impact of predictive genetic testing for Huntington's disease: a systematic review of the literature. J Genet Couns. 2015;24:29-39.

11. Wakefield CE, Hanlon LV, Tucker KM, et al. The psychological impact of genetic information on children: a systematic review. Genet Med. 2016;18:755-762.

12. Wynn J, Ottman R, Duong J, et al. Diagnostic exome sequencing in children: a survey of parental understanding, experience and psychological impact. Clin Genet. 2018;93:1039-1048.

13. Sanderson SC, Linderman MD, Suckiel SA, et al. Psychological and behavioural impact of returning personal results from whole-genome sequencing: the HealthSeq project. Eur J Hum Genet. 2017;25:280-292.

14. Green RC, Goddard KA, Jarvik GP, et al. Clinical Sequencing Exploratory Research Consortium: accelerating evidence-based practice of genomic medicine. Am J Hum Genet. 2016;98:1051-1066.

15. Gray SW, Martins Y, Feuerman LZ, et al. Social and behavioral research in genomic sequencing: approaches from the Clinical Sequencing Exploratory Research Consortium Outcomes and Measures Working Group. Genet Med. 2014;16:727-735.

16. Athens BA, Caldwell SL, Umstead KL, Connors PD, Brenna E, Biesecker BB. A systematic review of randomized controlled trials to assess outcomes of genetic counseling. J Genet Couns. 2017;26:902-933.

17. Berg JS, Amendola LM, Eng C, et al. Processes and preliminary outputs for identification of actionable genes as incidental findings in genomic sequence data in the Clinical Sequencing Exploratory Research Consortium. Genet Med. 2013;15:860-867.

18. Zigmond AS, Snaith RP. The hospital anxiety and depression scale. Acta Psychiatr Scand. 1983;67:361-370.

19. Kroenke K, Spitzer RL, Williams JB. The PHQ-9: validity of a brief depression severity measure. J Gen Intern Med. 2001;16:606-613.

20. Spitzer RL, Kroenke K, Williams JB, Lowe B. A brief measure for assessing generalized anxiety disorder: the GAD-7. Arch Intern Med. 2006;166:1092-1097

21. Cella D, Hughes $C$, Peterman A, et al. A brief assessment of concerns associated with genetic testing for cancer: the Multidimensional Impact of Cancer Risk Assessment (MICRA) questionnaire. Health Psychol. 2002;21:564-572.

22. Li M, Bennette CS, Amendola LM, et al. The Feelings About genomiC Testing Results (FACToR) Questionnaire: Development and Preliminary Validation. J Genet Couns. 2019:28:477-490.

23. DerSimonian R, Laird N. Meta-analysis in clinical trials. Control Clin Trials. 1986;7:177-188.

24. Stata Statistical Software [computer program]. Version 13.1. College Station, TX: StataCorp LP; 2013.

25. Broady KM, Ormond KE, Topol EJ, Schork NJ, Bloss CS. Predictors of adverse psychological experiences surrounding genome-wide profiling for disease risk. J Community Genet. 2018;9:217-225.

26. Vassy JL, Christensen KD, Schonman EF, et al. The impact of wholegenome sequencing on the primary care and outcomes of healthy adult patients: a pilot randomized trial. Ann Intern Med. 2017;167:159-169.

27. Lumish HS, Steinfeld H, Koval $C$, et al. Impact of panel gene testing for hereditary breast and ovarian cancer on patients. J Genet Couns. 2017;26:1116-1129.

28. Low CA, Bower JE, Kwan L, Seldon J. Benefit finding in response to BRCA1/2 testing. Ann Behav Med. 2008;35:61-69.

29. Krabbenborg L, Vissers LE, Schieving J, et al. Understanding the psychosocial effects of WES test results on parents of children with rare diseases. J Genet Couns. 2016;25:1207-1214. 
30. Rosell AM, Pena LD, Schoch K, et al. Not the end of the odyssey: parental perceptions of whole exome sequencing (WES) in pediatric undiagnosed disorders. J Genet Couns. 2016;25:1019-1031.

31. Malek J, Slashinski MJ, Robinson JO, et al. Parental perspectives on wholeexome sequencing in pediatric cancer: a typology of perceived utility. JCO Precision Oncology. 2017. https://doi.org/10.1200/PO.17.00010.

32. Sapp JC, Dong D, Stark C, et al. Parental attitudes, values, and beliefs toward the return of results from exome sequencing in children. Clin Genet. 2014;85:120-126.

33. Biesecker BB, Erby LH, Woolford S, et al. Development and validation of the Psychological Adaptation Scale (PAS): use in six studies of adaptation to a health condition or risk. Patient Educ Couns. 2013:93:248-254.

34. Christensen KD, Green RC How could disclosing incidental information from whole-genome sequencing affect patient behavior? Pers Med. 2013;10.
35. McBride CM, Koehly LM, Sanderson SC, Kaphingst KA. The behavioral response to personalized genetic information: will genetic risk profiles motivate individuals and families to choose more healthful behaviors? Annu Rev Public Health. 2010; 31:89-103.

36. Amendola LM, Berg JS, Horowitz CR, et al. The Clinical Sequencing Evidence-Generating Research Consortium: integrating genomic sequencing in diverse and medically underserved pPopulations. Am J Hum Genet. 2018;103:319-327.

37. Crawford DC, Crosslin DR, Tromp G, et al. eMERGEing progress in genomics—the first seven years. Front Genet. 2014;5:184.

38. All of Us. 2018. https://allofus.nih.gov/. Accessed 14 March 2018.

39. Berg JS, Agrawal PB, Bailey DB Jr, et al. Newborn sequencing in genomic medicine and public health. Pediatrics. 2017;139. 